\title{
Erratum: Atom-field dressed states in slow-light waveguide QED [Phys. Rev. A 93, 033833 (2016)]
}

\section{Giuseppe Calajó, Francesco Ciccarello, Darrick Chang, and Peter Rabl}

(Received 14 June 2016; published 24 June 2016)

DOI: 10.1103/PhysRevA.93.069905

In Sec. $\mathrm{V}$ of our original paper we have introduced the nonlinearity parameter

$$
\Delta_{\mathrm{nl}}\left(N_{e}\right)=\frac{\left|N_{e} E_{-}^{(1)}-E_{-}^{\left(N_{e}\right)}\right|}{g\left|N_{e}-\sqrt{N_{e}}\right|} .
$$

This parameter was then plotted in Fig. 9 for $N_{e}=2$ and the detunings $\delta=0, \delta=2 J$, and $\delta=3 J$. In all three cases the nonlinearity goes rapidly to zero for $g \lesssim J$. Although these results are correct, the plot shows only the nonlinearity parameters for the mostly photonlike states with $\delta=2 J$ and $\delta=3 J$, instead of the more relevant cases of the fully hybridized state at the band edge with $\delta=-2 J$ or the atomlike state inside the band gap with $\delta=-3 J$.

In Fig. 1 we show again the same plot of the nonlinearity parameter, but now including the cases $\delta=-2 J$ and $\delta=-3 J$ (we recall that in Fig. 9 we focus on the lowest dressed state). It can be seen that although for $\delta=-2 J$ the nonlinearity (compared to the Jaynes-Cummings nonlinearity) vanishes at small $g$, it is still much stronger than for the resonant case $\delta=0$. This is consistent with the observation that for $\delta=-2 J$ the wavelength of the second photon, $\lambda_{2}$, can be much larger than the wavelength of the first bound photon, $\lambda_{1}$ (see discussion in Sec. V C in the original paper). In contrast, for $\delta=0$ one finds $\lambda_{1} \approx \lambda_{2}$. Note that the approximate scaling of the nonlinearity parameter for $g \rightarrow 0$ can be understood from the simplified assumption $E_{-}^{(2)} \approx E_{-}^{(1)}-2 J$, which would correspond to a single-photon bound state plus an additional very loosely bound photon at the band edge. By recalling that $E_{-}^{(1)} \simeq-2 J-\left[g^{4} /(4 J)\right]^{1 / 3}$ [see Eq. (27) in the original paper], we obtain $\Delta_{\mathrm{nl}}(2) \sim \sqrt[3]{g}$.

For $\delta=-3 J$, which for $g \rightarrow 0$ corresponds to an atomlike state inside the band gap, the nonlinearity parameter diverges. Note that this divergence is a consequence of the chosen normalization for $\Delta_{\mathrm{nl}}\left(N_{e}\right)$ and can again be understood from the approximation $E_{-}^{(2)} \simeq \delta-2 J$ for small $g$.

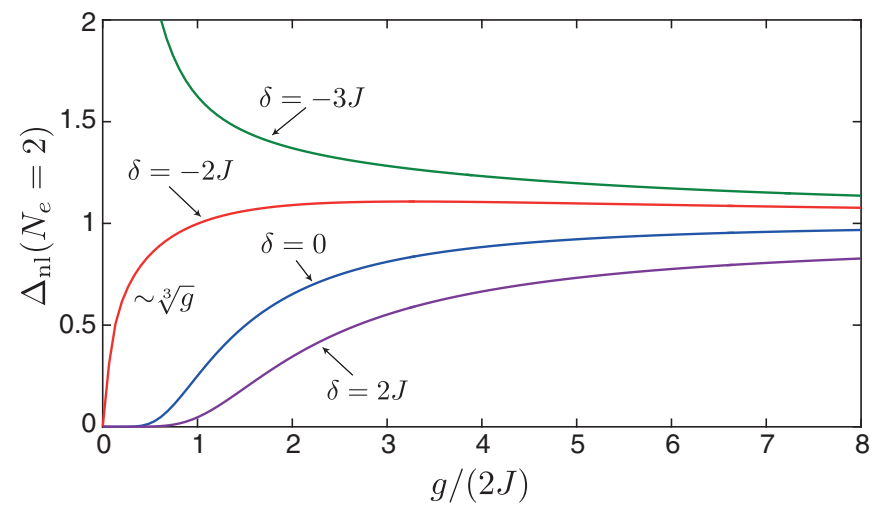

FIG. 1. Nonlinearity parameter $\Delta_{\mathrm{nl}}\left(N_{e}\right)$ as defined in Eq. (1) is plotted for $N_{e}=2$ and different atom-photon detunings $\delta$. 